\title{
LIMNOGRAFO A PRESSIONE
}

\author{
Francesco Peronaci
}

L'Istituto Nazionale di Geofisica ha intrapreso da qualche anno lo studio delle oscillazioni libere e forzate dei laghi italiani: i dati teorici già pubblicati non si sono spesso potuti confrontare con osservazioni di carattere sperimentale a causa della quasi assoluta mancanza del materiale di osservazione; pochissimi infatti, sono i laghi su cui esistono o siano esistiti degli impianti limnografici. Per ovviare a questa grave lacuna l'Istituto si è prefisso di far seguire alla fase teorica, delle campagne di misure oltre ad istallare degli strumenti che permettano delle osservazioni sistematiche. Il compito è arduo in quanto esso richiede oltre al rilevamento dei dati meteorologici (vento, pressione) l'impianto per ogni lago di un numero notevole di limnografi allo scopo di verificare i periodi delle sesse nelle varie zone del bacino, la posizione delle linee nodali, ventrali, la presenza di eventuali sesse simmetriche. L'istallazione di un limnografo richiede spesso, per la natura stessa delle sponde del lago e del fondale, l'esecuzione di lavori preliminari spesso in muratura nonché la costruzione di pontili, palafitte ecc.; in quanto non sempre il punto più intercssante per la registrazione si presta naturalmente alla sistemazione dell'apparecchio. Per ovviare a questo inconveniente, mi sono prefisso di costruire uno strumento che sia particolarmente adatto ad una campagna di rilevamenti in cui sia necessario eseguire molte misure in più luoghi e contemporaneamente, e che possa porsi in funzione con qualunque fondale senza l'esecuzione di sistemazioni preliminari. Ho cercato altresì di diminuire il più possibile le dimensioni di ingombro e il peso per facilitare il trasporto sul luogo d'osservazione, ed ho ridotto al minimo gli organi di trasmissione escludendo puleggie ed ingranaggi per evitare quei giuochi che si verificano inevitabilmente nei modelli a galleggiante esistenti in commercio.

Lo strumento è rappresentato schematicamente nella figura 1; esso si compone di un registratore, di un sistema per segnare il tempo e di una parte sensibile. Il registratore $(B)$ è del tipo normale ad orologeria con 36 ore di carica e uno scorrimento di $3 \mathrm{~mm}$ al minuto primo. La registrazione del tempo è affidata ad un orologio che co- 


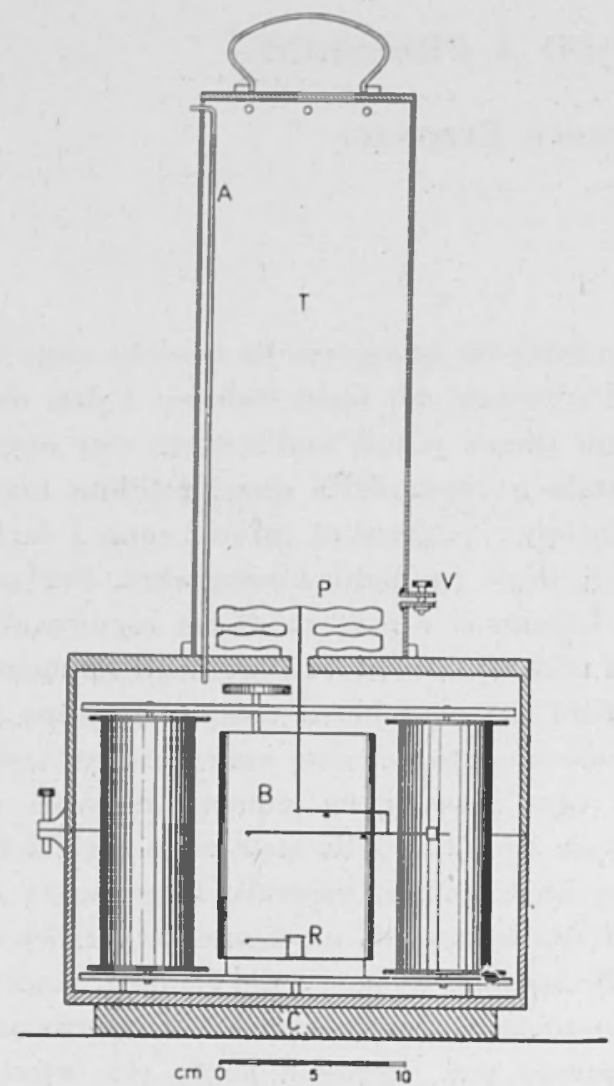

Fig. 1

manda una elettrocalamita $(R$ ) alimentata da una pila a secco; il tempo viene segnato ogni 15 minuti sul diagramma mediante un forellino.

La parte sensibile consta di un polmone $(P)$, simile a quello in uso nei barometri aneroidi, posto in comunicazione con l'interno dello strumento ed azionante una pennina attraverso un complesso di leve che danno luogo ad un ingrandimento regolabile a piacere. Tutto lo strumento è racchiuso in una cassetta cilindrica di alluminio appesantita da una base di piombo; dalla cassetta fuoriescono solo il polmone ed un piccolo tubo (A) che mantiene la pressione all'interno dello strumento allo stesso valore della pressione atmosferica. II limnografo viene immerso in acqua ad una profondità di 50)-70 $\mathrm{cm}$ in modo clie il polmone registri le variazioni di pressione generate dalla sessa. Per eliminare la registrazione del moto ondoso, la parte sensibile è circondata da un cilindro $(T)$, posto in comunicazione con la libera atmosfera, che permette l'ingresso dell'acqua attraverso una valvola $(V)$, che viene opportunamente regolata a seconda del periodo della sessa da rilevare.

Un primo modello, costruito dall'officina dell'I.N.G., lıa già funzionato per vari giorni sul lago di Bracciano con esito più che soddisfacente. In figura è riportato un esempio delle registrazioni ottenute (onda uninodale), esse sono pir̀ nitide agli effetti della determinazione dei periodi delle analoghe ottenute con un limnografo a galleggiante che funzionava contemporaneamente.

Lo strumento, diminuendo opportunamente lo scorrimento e re- 

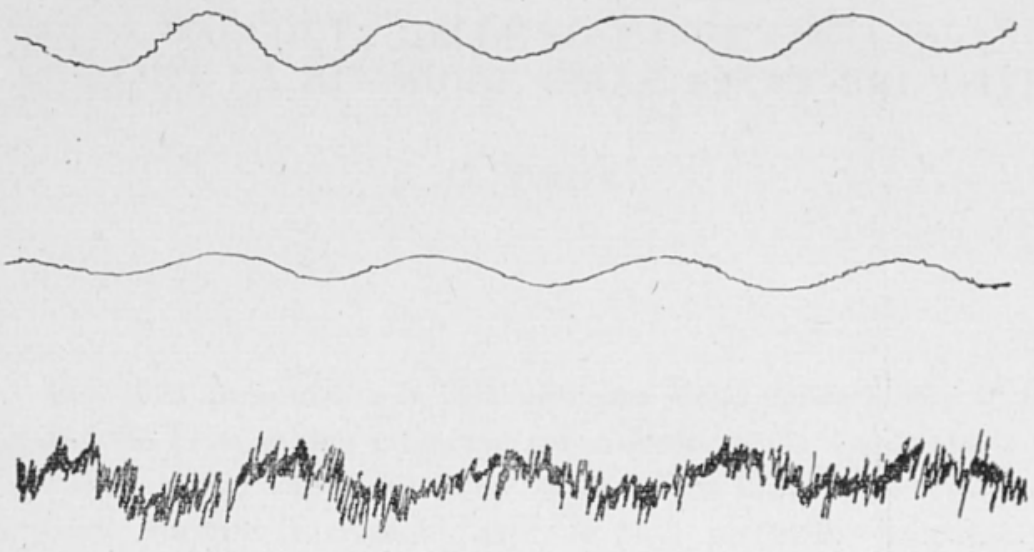

Iig. 2

golando la valvola d'ingresso del pozzetto, può essere usato anche per la registrazione delle lente variazioni di livello e dell'effetto in superficie delle sesse termiche.

Roma - Istituto Nazionale di Geofisica - Gennaio 1950.

\section{RIASSUNTO}

Si descrive un nuovo tipo di limnografo a pressione portatile particolarmente adatto per campagne di rilevamenti, e si riportano al. cune registrazioni ottenute con esso sul lago di Bracciano. 